\title{
Human-specific CpG 'beacons' identify human-specific prefrontal cortex H3K4me3 chromatin peaks
}

\begin{abstract}
Background: Targeted recruitment of chromatin-modifying enzymes to clusters of $\mathrm{CpG}$ dinucleotides contributes toward the formation of accessible chromatin. By interprimate comparison we previously identified the set of nonpolymorphic humanspecific CpGs (CpG 'beacons') and revealed that these loci were enriched for human disease traits. Due to their human-specific CpG density change, extreme CpG 'beacon' clusters ( $\geq 20 \mathrm{CpG}$ beacons $/ \mathrm{kb}$ ) were predicted to identify permissive chromatin peaks within the human genome. Aim: We set out to explore these sequence-defined regions for evidence of an active chromatin signature. Results: Using available comparative primate epigenomic data from neurons of the prefrontal cortex, we show that these CpG 'beacon' clusters are indeed enriched for being human-specific H3K4me3 peaks $\left(\chi^{2}: p<2.2 \times 10^{-16}\right)$ and thus predictive of permissive chromatin states. These sequence regions had a higher predictive value than previous selective analyses. We also show that both human-specific H3K4me3 and CPG 'beacon' clusters are increased within current and ancestral telomeric regions, supporting an association with recombination, which is higher towards the distal ends of chromosomes. Conclusion: Therefore, CpGfocused comparative sequence analysis can precisely pinpoint chromatin structures that contribute to the human-specific phenotype and further supports an integrated approach in genomic and epigenomic studies.
\end{abstract}

Keywords: chromatin $\bullet$ comparative epigenomics $\bullet$ CpG islands $\bullet$ DNA methylation - epigenetics $\bullet$ epigenomics $\bullet$ gene regulation $\bullet$ human evolution $\bullet$ primate epigenetics

\section{Background}

Clusters of $\mathrm{CpG}$ dinucleotides, termed $\mathrm{CpG}$ islands (CpGis), act as a genomic platform for gene expression by providing an accessible environment for the binding of the basal transcription machinery $[1,2]$. They facilitate this setting, due to both their distinctive density and predominately unmethylated state, by recruiting specific factors that modify the three dimensional structure of the local chromatin $[3,4]$. Hence these islands reside within an expanse of repressed genome, forming active or transcriptionally competent promoters [5] and possess the characteristic permissive $\mathrm{H} 3 \mathrm{~K} 4 \mathrm{me} 3$ histone signature [6]. This chromatin configuration is itself associated with expression, but also may further enable the recruitment of components required for this activity $[7,8]$.
Due to this direct effect on 3D chromatin structure by $\mathrm{CpG}$ dinucleotides [5], and the potential for regulatory modulation it creates, we previously examined the primate-alignable portion of human genome for nucleotide sequence change that has led to the formation or maintenance of human-specific CpGs [9]. This was performed by sequence comparison of six primates (Enredo-Pecan-Ortheus [EPO] alignment including: human, chimpanzee, gorilla, orangutan, rhesus macaque and marmoset $[10,11])$. The resultant nonpolymorphic human-specific CpGs were termed human CpG 'beacons' and identify prospective novel human-specific regulatory regions. These CpG 'beacons' are now shown to be markedly enriched in the dynamic portion of the human DNA methylome [12]. We then
Christopher G Bell*:,2, Gareth A Wilson' \& Stephan Beck ${ }^{1}$ ${ }^{1}$ Medical Genomics, UCL Cancer Institute, University College London, London, UK

${ }^{2}$ Current Address: Department of Twin Research \& Genetic Epidemiology, St Thomas' Hospital, King's College London, London, UK

*Author for correspondence: christopher.g.bell@kcl.ac.uk

Future
Medicine $\mathrm{fS}$ 
identified 21 regions of extreme $\mathrm{CpG}$ 'beacon' clusters (empirical $\mathrm{p}<1 \times 10^{-3}$ ) within the human genome. These were shown to colocate with four causative genes in monogenic mental/developmental disorders (ANKRD11 [13], CHL1 [14], EHMT1 [15] and VLDLR $[16,17])$, and genes implicated with complex traits such as autism and psychiatric disease (ANKRD11 [18], DLGAP2 [19] and DPP10 [18,20]), as well as reidentifying the noncoding RNA, HAR1A [21], implicated in cortex development, by precisely hitting its CpGi. These loci were significantly statistically enriched for neurological trait-related genes. As methylation is inversely correlated with CpGi density [22,23], we were also able to indicate the functional consequences of species-specific $\mathrm{CpG}$ density change, through comparative DNA methylome analysis in human, chimpanzee and macaque, with expected changes in methylation in orthologous CpGi.

A recently published comparative chromatin analysis for human-specific H3K4me3 within the prefrontal cortex (PFC) between human, chimpanzee and macaque identified 410 loci with a human-specific peak gain [24]. This dataset provided the ideal opportunity to test whether the hypothesized structural effect of CpG 'beacon' clusters did in fact lead to humanspecific chromatin modification. Furthermore, these data are derived from a cortical region fundamental in human-derived higher functioning [25,26].

Therefore, we tested this hypothesis and identified that CpG 'beacon' clusters are indeed enriched for these human-specific accessible chromatin regions. Furthermore they are increasingly likely to precisely locate the most significant fraction of these peaks. This supports the idea that human species-specific CpG 'beacons' have played a critical role in human evolution, as they locate significant regions of humanspecific functional epigenomic change in the genome, and that $\mathrm{CpG}$-focused comparative sequence analysis between the closely related primate family can be highly informative.

\section{Methods}

Data

Human-specific H3K4me3 PFC peak locations were taken from the supplementary data (Supplementary Table S3) of Shulha et al. [24].

Location of human Chip-seq H3K4me3 PFC peaks were accessed from Maunakea et al. [27], via the UCSC Genome Browser Table Browser: Regulation; UCSF Brain DNA Methylation; ucsfChipSeqH3K$4 \mathrm{me} 3 \mathrm{BrainC}$ Coverage. The sequencing reads are available through the NCBI SRA (study accession number SRP002318) [28]. The CpG 'beacon' data is browser viewable within UCSC [29].

\section{Analysis}

All statistical calculations and simulations were performed in $\mathrm{R}$ [30]. Wilcoxon test was used for hsH3K4me3 and CpG 'beacon' comparison between loci with $0 \mathrm{CpG}$ 'beacons' and those with $\geq 20$, using the FDR p-values calculated by Shulha et al. for human versus chimpanzee and macaque regions [24]. For this FDR comparison analysis, those loci with an FDR $\mathrm{p}=0$ were capped at a $\mathrm{p}=1 \times 10^{-200}$. Genome intersect comparisons were via the BEDTools package command intersectBed [31]. Genomic representation plot was constructed with Circos [32]. Genomic enrichment calculations performed with GREAT, by the region-based binominal method, with regional default for associated genomic regions, but with extension reduced to $100 \mathrm{~kb}$ [33]. A locus was considered to reside in a distal, or telomeric region, if it overlapped the first or last chromosome band (coordinates via Ensembl Biomart). All human genomic coordinates given are in the GRCh37/hg19 build.

\section{Results}

Primate comparative sequence analysis predicts human-specific chromatin state

A set of 410 human-specific peaks of the activating chromatin modification, H3K4me3, were identified from a total of 34,639 human peaks, by a comparative analysis of the PFC of human, chimpanzee and macaque by Shulha et al. [24]. Within the 21 extreme CpG 'beacon' peak regions we previously identified [9], available data from Maunakea et al. had shown that 8 possessed H3K4me3 PFC peaks [9,27]. Comparison of these 8 peaks with the Shulha et al. dataset revealed 5 to be identified as human-specific H3K4me3 (hsH3K4me3) peaks. Therefore the H3K4me3 signal in these CpG 'beacon' clusters is strongly enriched for being present only in human and not in chimpanzee or macaque $\left(\chi^{2}: p<2.2 \times 10^{-16}\right)$. These five hs-H3K4me3 peaks were precisely located by extreme $\mathrm{CpG}$ 'beacon' clusters ( $\geq 20 \mathrm{CpG}$ 'beacons' $/ \mathrm{kb}$ ) within or near the genes; ANKRD11, CHL1, DPP10, HAR1A and PSD4 (see Figures 1 \& 2 \& Supplementary Figure 1; accessible online at www.futuremedicine.com/doi/ suppl/10.2217/epi.13.74).

Randomization via ShuffleBed [31] and genome structure correction (GSC) enrichment [34,35] were highly significant (both $\mathrm{p}<1 \times 10^{-5}$ ). However, both the extreme CpG 'beacon' clusters and H3K4me3 peaks are not likely to be found with equal chance throughout the entire genome, but are highly likely to reside within $\mathrm{CpG}$-dense regions. To account for this, we permutated the 21 loci, representing random extreme CpG clusters, only from the total of 18,665 human PFC H3K4me3 peaks, out of the total of 
37,902 (identified from Maunakea et al. [27], as the total set genome co-ordinates are not available from Shulha et al. [24]) that overlap CpG-dense regions (22,374 Ensembl CpGis). With 10,000 permutations this did not exceed the observed five peaks (average: $0.466 \pm 0.677$ overlapping peaks per genome simulation, empirical $\left.\mathrm{p}<1 \times 10^{-5}\right)$. Therefore while as much as $\sim 83.4 \%$ of brain PFC CpGi's possess the H3K4me3 signature, only $\sim 1.8 \%$ have human-specific $\mathrm{H} 3 \mathrm{~K} 4 \mathrm{me} 3$, whereas by contrast, the $\mathrm{CpG}$ 'beacon' clusters are statistically strongly enriched with $\sim 23.8 \%$ having human-specific H3K4me3 (five out of 21 clusters).

\section{Further statistical overlap at lower CpG cluster} thresholds

CpG 'beacon' H3K4me3 overlaps were not only restricted to the most extreme clusters $(\geq 20 \mathrm{CpG}$ 'beacons'/kb) but also found at lower levels. At a CpG 'beacons' density level of $15 / \mathrm{kb}, 11$ out of a total of 63 cluster regions overlap with hs-H3K4me3 (simulation average: $1.387 \pm 1.164$ ) and 20 out of a total of 254 cluster regions overlaps at $\mathrm{CpG}$ 'beacons' density level of $10 / \mathrm{kb}$ (simulation average: $5.604 \pm 2.347$ ). Permutation analysis therefore for both of these results are highly statistically unlikely (all empirical $\mathrm{p}<1 \times 10^{-5}$ ).

When we initially calculated the set of the extreme CpG 'beacon' clusters we took a conservative genomewide empirical cut-off of $\mathrm{p}<1 \times 10^{-3}$, which was reached at a density level of $20 / \mathrm{kb}$ CpG 'beacons'. The permutation however does not become nonsignificant ( $p>0.05)$ until a density level of $<17 / \mathrm{kb}(\mathrm{p}=0.210)$ and furthermore even at the $17 / \mathrm{kb}$ level the binominal genomic enrichment results via GREAT [33] for cognition is significant $\left(\mathrm{p}=7.6 \times 10^{-6}, \mathrm{FDR} Q=3.36 \times 10^{-2}\right)$. The H3K4me3 peak overlap at $17 / \mathrm{kb}$ is 7 , out of a possible 36, (empirical $\mathrm{p}<1 \times 10^{-5}$, see Table 1). While the lower CpG 'beacon' density levels of $15 / \mathrm{kb}$ and $10 / \mathrm{kb}$ are not statistical outliers in their own right, this still does not negate their potential functional importance, and the enrichment for hs-H3K4me3 in these loci strongly supports this.

Additionally, the defined CpG 'beacons' were identified only within the $\sim 80 \%$ of the human genome with rigorous primate homology from the EPO sequence block alignments $[10,11]$ that included, at least, human, chimpanzee and one other primate sequence (abbreviated as the 'h1clol' genome). Of the total 410 hs-H3K4me3 peak regions, only 257 lie within this majority of the genome, therefore, an under-representation ( $20 \%$ loss genome but $34.15 \%$ reduction in peaks; $\left.\chi^{2}: \mathrm{p}<2.2 \times 10^{-16}\right)$. Thus the primate nonhomologous regions contain more identified hs-H3K4me3 peaks. This may be due to stronger genetic divergence within these regions having a con-

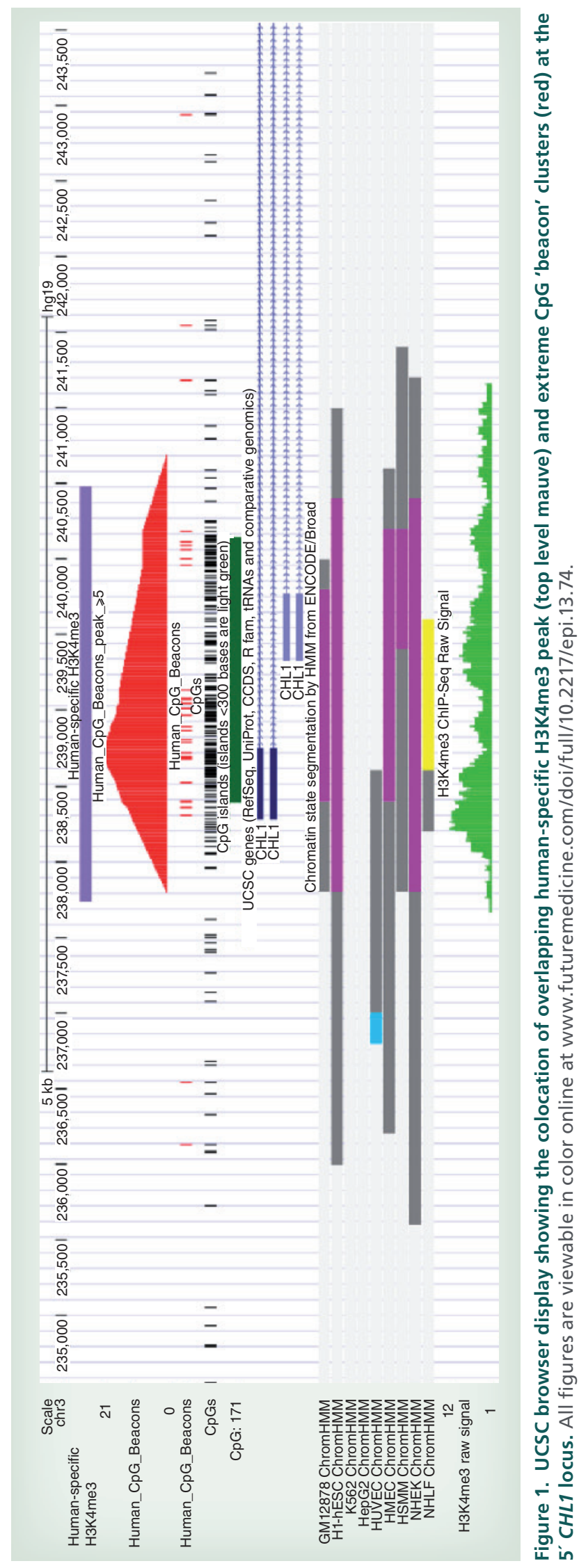




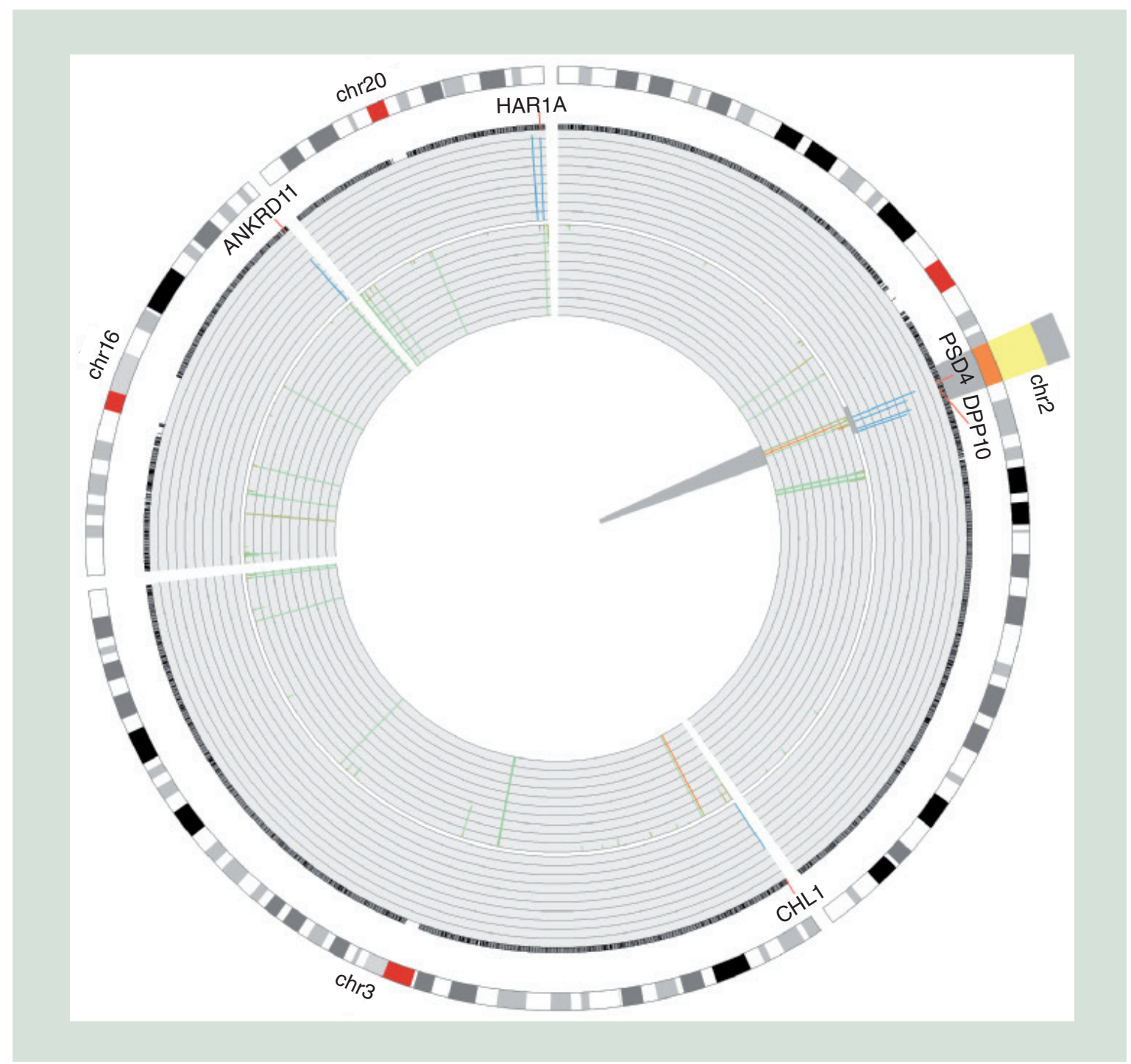

Figure 2. Human chromosomes 2, 3, 16 and 20. Outer ring: chromosomes with banding patterns. Dense edge ring: Enredo-Pecan-Ortheus $[10,11]$ h1c101 coverage of these chromosomes. Blue peaks: extreme CpG 'beacon' peak locations. Human-specific H3K4me3 location with false discovery rate log p-values compared to chimpanzee (orange), macaque (green) and overlapping chimpanzee and macaque (olive). Location of ANKRD11, CHL1, DPP10, HAR1A and PSD4 indicated with colocating CpG 'beacon' clusters and human-specific H3K4me3. The highlighted yellow wedge is the location of the chromosome 2qFus (2q13-2q14.1) with enrichment of both extreme CpG 'beacon' clusters and of human-specific H3K4me3 peaks $\left(\chi^{2}: p=1.045 \times 10^{-5}\right)$.

sequential stronger influence on the epigenome, but also could be contributed to by a higher potential analytical bias component. $30(\sim 12 \%)$ of these 257 peaks overlap with a CpG beacon peak of at least $\geq 5 / \mathrm{kb}$.

\section{Predicted H3K4me3 peaks have the strongest evidence for a human-specific state}

By utilizing the FDR p-value calculated by Shulha et al. for their $\mathrm{H} 3 \mathrm{~K} 4 \mathrm{me} 3$ peaks [24], we find that the most extreme CpG 'beacon' clusters that overlap with hs-H3K4me3 peaks have a significantly higher likelihood of being truly human-specific peaks. These loci have more significant FDR p-values in comparison with both potential chimpanzee and macaque loci (loci with $\mathrm{CpG}$ 'beacons' $=0$, compared with those with $\geq 20$, Wilcoxon $\mathrm{p}=3.57 \times 10^{-3}$ for human vs chimpanzee and $\mathrm{p}=2.50 \times 10^{-2}$ for human vs macaque, see Figure 3A \& B). Consequently, while the 410 peaks have variable certainty as to being truly human-specific, as represented by these FDR values, the most likely hs$\mathrm{H} 3 \mathrm{~K} 4 \mathrm{me} 3$ lie within extreme CpG clusters. Therefore the conservatively calculated enrichment within these hs-H3K4me3 would be even stronger if false-positive hs-H3K4me3 peaks were excluded. Additional datasets from the other closely related primates, including orangutan and gorilla, would aid the reduction in 


\begin{tabular}{|c|c|c|c|c|c|c|c|c|}
\hline 32 & $\begin{array}{l}\text { chr20:61732970- } \\
61734710\end{array}$ & $H A R 1 B$ & $5^{\prime}$ promoter & 0 & $5.302 \times 10^{-10}$ & $0.00 \times 10^{+00}$ & Yes & $\begin{array}{l}\text { *610556/ } \\
\text { *610557 }\end{array}$ \\
\hline 26 & $\begin{array}{l}\text { chr2:113914422- } \\
113915745\end{array}$ & PSD4 & Upstream & 15,814 & $4.101 \times 10^{-9}$ & $4.670 \times 10^{-11}$ & Yes & *614442 \\
\hline 22 & $\begin{array}{l}\text { chr2:115419442- } \\
115420562\end{array}$ & $\begin{array}{l}\text { DPP10/ } \\
\text { LOC389023 }\end{array}$ & Intragenic & 200,263 & $1.340 \times 10^{-38}$ & $0.00 \times 10^{+00}$ & Yes & *608209 \\
\hline 21 & $\begin{array}{l}\text { chr3:237734- } \\
240480\end{array}$ & $\mathrm{CHL1}$ & $5^{\prime}$ promoter & 0 & $2.296 \times 10^{-42}$ & $3.501 \times 10^{-92}$ & Yes & $\begin{array}{l}\text { *607416/ } \\
\# 613792\end{array}$ \\
\hline 21 & $\begin{array}{l}\text { chr16:89322850- } \\
89324461\end{array}$ & $\begin{array}{l}\text { ANKRD11/ } \\
\text { ZNF778 }\end{array}$ & $3^{\prime}$ & 38,740 & $1.239 \times 10^{-27}$ & $0.00 \times 10^{+00}$ & Yes & $\begin{array}{l}* 611192 / \\
\# 148050\end{array}$ \\
\hline 17 & $\begin{array}{l}\text { chr2:115918003- } \\
115921686\end{array}$ & DPP10 & $5^{\prime}$ promoter & 0 & $7.824 \times 10^{-29}$ & $1.046 \times 10^{-13}$ & Yes & *608209 \\
\hline
\end{tabular}

false-positive human-specific peaks, however these are with an approximately two- to three-fold enrichment. currently not publicly available.

Double peaks \& telomeric bias

Peaks of hs-H3K4me3 were also identified by Shulha Recent ENCODE data has identified an average peak region of long range interaction of promoters, enhancers and CTCF to be approximately $120 \mathrm{~kb}$ upstream of the TSS [36]. This grouping propensity is also seen et al. to occur in pairs or groups within $0.5-1 \mathrm{Mb}$, within the $\mathrm{CpG}$ 'beacon' overlap peaks (i.e., DPP10

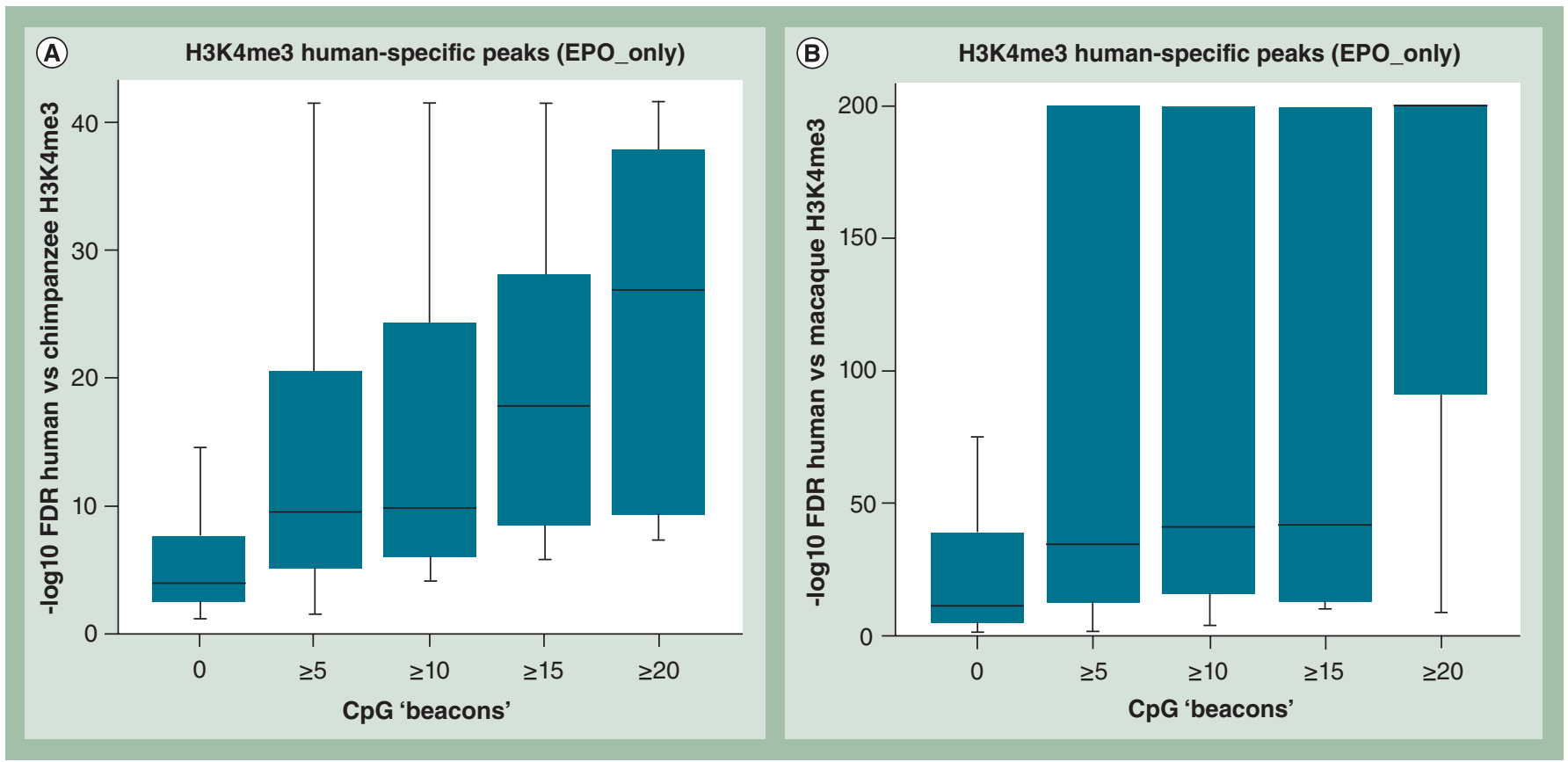

Figure 3. Stronger human-specific H3K4m3 peaks identified (FDR p-value) with CpG 'beacon' density in both human versus chimpanzee (A), and versus rhesus macaque (B) (Wilcoxon test CpG 'beacon' 0 vs $\geq 20, p=3.57 \times 10^{-3}$ chimpanzee and $p=2.50 \times 10^{-2}$ macaque $\left[p=0\right.$ values capped at $\left.\left.10^{-200}\right]\right)$. This analysis is within the primate-alignable $(E P O[10,11])$ portion of the human genome. EPO: Enredo-Pecan-Ortheus; FDR: False discovery rate. 
and EXOC2, among others; Supplementary Table 1). These double peaks had an average genomic distance between them of $\sim 126 \mathrm{~kb}$. Shulha et al. showed evidence of these close double peak regions having physical interaction by ChIA-PET. ChromHMM segmentation analysis [37] found that colocating with $\mathrm{H} 3 \mathrm{~K} 4 \mathrm{mel}$, a high level of H3K4me3 (61-80\%) was also in Strong Enhancer Type 4, thus potentially implicating some of these regions in this role.

We previously identified a distal chromosome or telomeric bias in CpG 'beacon' clusters (CpG 'beacon' cluster $20 / \mathrm{kb}=52.3 \%$ in terminal chromosome bands [9]), due to the role recombination-associated biased gene conversion (BGC) plays in their formation. This being the biochemical bias in the DNA repair mechanism that occurs in heteroduplexed DNA, formed during crossing over, leading to clustered weak-to-strong 'biased' substitutions (AT to GC) [38,39]. Therefore, we assessed whether hs-H3K4me3 also have a telomeric bias which could be contributing to these peak groupings. We calculated there is in fact a >twofold enrichment within these telomeric regions compared to all human H3K4me3 peaks $\left(\chi^{2}: \mathrm{p}<2.2 \times 10^{-16}\right)$.

We also previously identified a strong clustering bias of CpG 'beacon' clusters, within the historic chromosome $2 \mathrm{qFus}$ region, due to past recombination in these archaic telomeric regions [40]. Examining the hsH3K4me3 peaks, these also show an enrichment for this 2qfus region (2q13-2q14.1 - chr2: 110,200,001$118,800,000$ ) with an average 0.699 peaks/Mb compared with $\sim 0.131$ peaks $/ \mathrm{Mb}$ across the genome and an approximately sixfold enrichment over the expected hsH3K4me 3 compared to all the human $\mathrm{H} 3 \mathrm{~K} 4 \mathrm{me} 3$ peaks present here $\left(\chi^{2}: p=1.045 \times 10^{-5}\right.$; see Figure 2$)$. Hence this further supports the model of recombination-associated BGC increase in $\mathrm{CpGs}$ leading to the formation of $\mathrm{H} 3 \mathrm{~K} 4 \mathrm{me} 3$ peaks. Cohen et al. also recently proposed BGC islands, as one of the categories of CpG islands, due to their formation by this process [41].

\section{CpG 'beacons' predict human-specific H3K4me3 peaks greater than other comparative sequence change}

Shulha et al. compared the identified hs-H3K4me3 peaks with known regions of accelerated humanspecific sequence change, such as the human accelerated regions (HARs) [21] and found only minimal overlap $(1 / 49$ loci $=H A R 1 A)$, as well as nine further studies of positive selection collated together by Akey [42], which also showed low intersection. Due to this inadequate overlap they concluded that comparative sequence analysis was poorly informative of potential functional epigenomic differences [24] and thus that important changes in chromatin structure and func- tion cannot be identified by comparative genomic analysis alone. Furthermore, we also compared the hs-H3K4me3 regions with the human accelerated conserved noncoding (HACNS - 0/992 loci) [43] and accelerated noncoding (ANC - 6/1356 loci) [44] sets and these also show minimal colocation. However, as shown, the specific CpG dinucleotide change, indicated by $\mathrm{CpG}$ 'beacons' through six primate comparison, enabled a far better prediction of hs-H3K4me3 than any of these methods. Therefore important changes in chromatin structure and epigenomic function can be identified by specific comparative genomic analysis.

We simulated these data by permutation with random selections sets of one to 1000 loci from the total of 18,665 human peaks $(1000 \times$ randomization each) that then overlap the $410 \mathrm{hs}-\mathrm{H} 3 \mathrm{~K} 3 \mathrm{me} 3$ and plotted these results on a scatter plot also with the observed overlaps for all these mentioned studies (blue squares) and the CpG 'beacon' data (red and green Squares) (see Figure 4). This clearly shows that while the selection studies perform either the same or worse than the simulation values, the $\mathrm{CpG}$ 'beacon' clusters overlap is far in excess of the maximum simulation results (all empirical $\mathrm{p}<1 \times 10^{-5}$ ), with those adjusted for the reduced numbers within the primate EPO alignment performing the best (green squares).

The only study that approaches the success of the CpG 'beacons' is the expanded set of 202 HARs $(\sim 85.6 \mathrm{kB})$, as while the top 49 HARs perform poorly, when this set is extended further these loci begin to capture more $\mathrm{CpG}$ 'beacon' clusters, such that $\sim 85.7 \%$ of the hs-H3K4me3-overlapping HAR_202 regions are CpG 'beacon' clusters of $\geq 10 / \mathrm{kb}$ (12 out of 14). This overlap, as discussed previously, indicates the strong role of BGC in the formation of both $\mathrm{CpG}$ 'beacon' clusters and regions of extreme species-specific dinucleotide divergence in the human genome, driven by recombination $[9,38]$. Thus, further indicating how this process, with a by-product of modulating sequence, can affect genomic $3 \mathrm{D}$ structure in these extreme locations.

The simulation also clearly displays why the comparison against the 9 positive selective studies as used by Shulha et al. [24] is inappropriate to identify human-specific change, as these studies use human population variation and linkage disequilibrium data that is too recent to identify significant genetic changes that have led to variation between primates. Furthermore, the other comparative studies lose power in comparison with the 'beacon' analysis, as they regard all nucleotide change as equal, are focused wider than an interprimate analysis, and in those that center exclusively on nontranscript over- 
lapping sequence, such as the ANC regions [44], may exclude many promoter hs-H3K4me3 regions that reside $5^{\prime}$ within first exons, or intragenic promoter CpGi loci. The inaccuracy of using human population data can be starkly seen by the fact that the genome space associated with two or more selective studies from Akey is $244.7 \mathrm{Mb}$, compared with only $29.9 \mathrm{~kb}$ for the extreme CpG 'beacon' $\geq 20 / \mathrm{kb}$ peaks regions, or $\sim 0.01 \%$ of this, and yet the latter has $23.8 \%$ successful overlap with hs-H3K4me3 peaks versus $2.36 \%$ of the former.

\section{Discussion}

DNA methylation is essential for mammalian development and is almost exclusively restricted to the CpG dinucleotide in differentiated cells [45]. Therefore, this epigenomic mechanism requires the presence of the $\mathrm{CpG}$ genetic sequence template in order to facilitate the modification's dynamic functionality. Although a lower level of non-CpG cytosine methylation has been identified to occur in the human brain, predominately at CACC motifs [46]. We previously identified, by interprimate comparative analysis, human-specific CpG 'beacon' clusters and found these to be enriched for human disease and phenotype loci. The facilitated epigenetic role that these clusters of $\mathrm{CpGs}$ provide is not restricted to DNA methylation, but may also be involved in enabling other modifications of DNA, including hydroxymethylation [47]. Furthermore, we predicted that these clusters would influence chromatin structure, due to their significant human-specific change in CpG density. Using the PFC hs-H3K4me3 data from Shulha et al. [24] we have shown strong evidence of this. This sequence relationship is only associated with $\mathrm{H} 3 \mathrm{~K} 4 \mathrm{me} 3$, and presently only tested in PFC, but further analysis, when other comparative epigenomic tissue signatures become available, may be revealing.

Mechanisms have evolved to reduce the local functional impact of transposable elements, such as Alu elements [48], which while possessing high CpG content, are usually methylated [49]. However sequence divergence of homologous loci through highly dissimilar species-specific recombination [50], driven by the rapidly evolving recognition motif of PRDM9 [51,52], can lead to BGC-associated increase in local GC content [53]. This can concurrently increase $\mathrm{CpG}$ dinucleotides and form increasingly dense [54] or new $\mathrm{CpGi}$, within these hotspots [55], even without requiring the force of positive selection [41]. These CpGi regions, recruit chromatin-modifying enzymes, such as Cfp1, as has been experimentally shown by $\mathrm{CpG}$ island inclusion experiments in a mouse model by Thomson $e t$ al. [3]. This, in turn, leads to the targeted formation of acces-

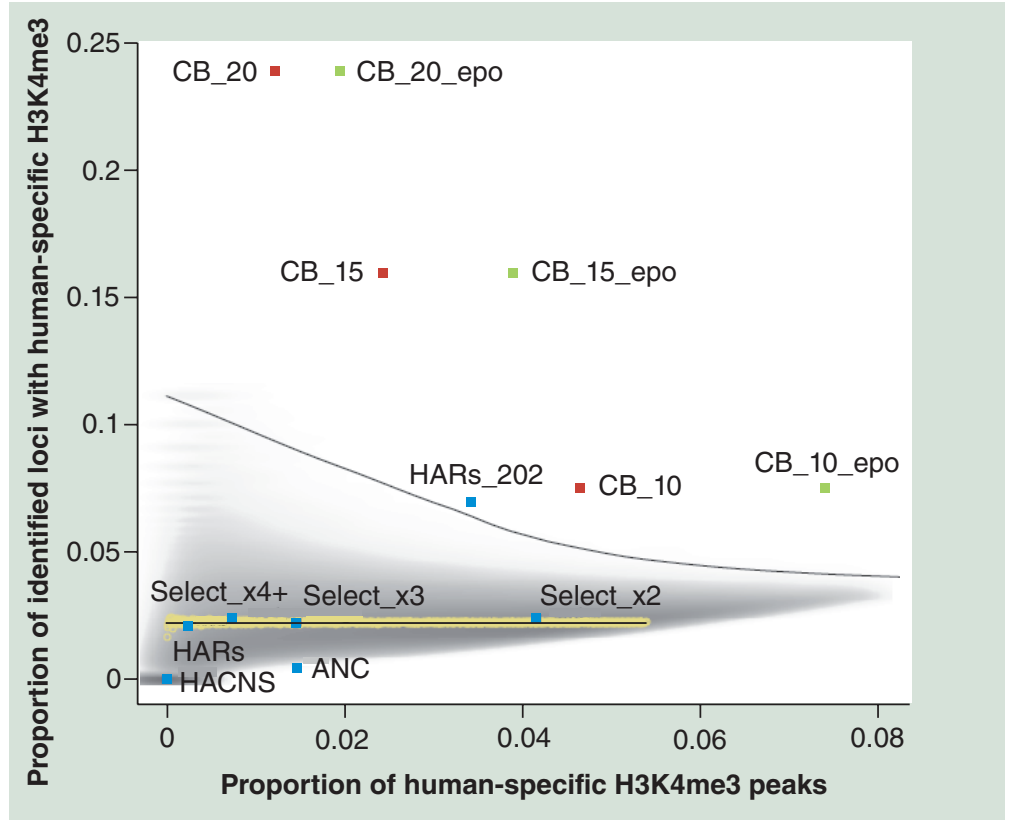

Figure 4. Comparison of studies: proportion of identified loci with overlapping human-specific H3K4me3. CpG 'beacon' clusters identify hs-H3K4me3 peaks more successfully than extreme human nucleotide change or studies of selection. Scatter plot of permutated data for random selection of sets of one to 1000 loci from the total of 18,665 human peaks ( $1000 \times$ randomization each) that then overlap the 410 hs-H3K3me4 loci. Upper line: Loess regression line of maximum simulation value; golden points: average for each random selection with average Loess regression line overlaid in grey; red squares: CpG 'beacon' clusters; green squares: CpG 'beacon' clusters adjusted for the fact that the primate EPO alignment only contains $257 \mathrm{hs}-\mathrm{H} 3 \mathrm{~K} 4 \mathrm{me} 3$ peaks; blue squares: selective studies - HACNS (HACNS - 0/992) [43] and ANC (ANC 6/1356) [44], Select_x2,_x3 and_x4+ are overlaps with studies combined by Akey [42] with loci colocating in 2, 3 or 4 or more studies. HARs are the 49 top HARs [21]. HAR_202 is an expanded set of 202 HARs, with this set the only nonbeacon analysis to perform well, as this larger grouping has a strong overlap with CpG 'beacons' ( $85.7 \%$ of the hs-H3K4me3 overlapping CpG 'beacons' $\geq 10 / \mathrm{kb}$ ).

ANC: Accelerated noncoding; $C B \_10$ : CpG 'beacon' clusters $\geq 10 / \mathrm{kb}$;

CB_15: CpG 'beacon' clusters $\geq 15 / k b$; $C B \_20$ : CpG 'beacon' clusters $\geq 20 / k b$; HAR: Human accelerated regions; HACNS: Human accelerated conserved noncoding; hs-H3K4me3: Human-specific H3K4me3.

sible H3K4me3 chromatin structure in the appropriate genomic regions [56]. This chromatin signature may not itself be causative in expression, but is indicative of a permissive promoter formation. Furthermore independent of transcription status, both CpG content and $\mathrm{CpGi}$ width correlate with local nucleosome depletion [57].

Dreszer et al. has previously identified outlying regions of high BGC with loci of extreme sequence diverge, and these regions also have a telomeric or distal bias, due to the higher level of recombination that occurs there $[38,58]$. CpG 'beacon' clusters also share these properties [9] and thus link recombination with H3K4me3 formation (see Hypothesis in Figure 5). Therefore the extreme $\mathrm{CpG}$ increase identified in 


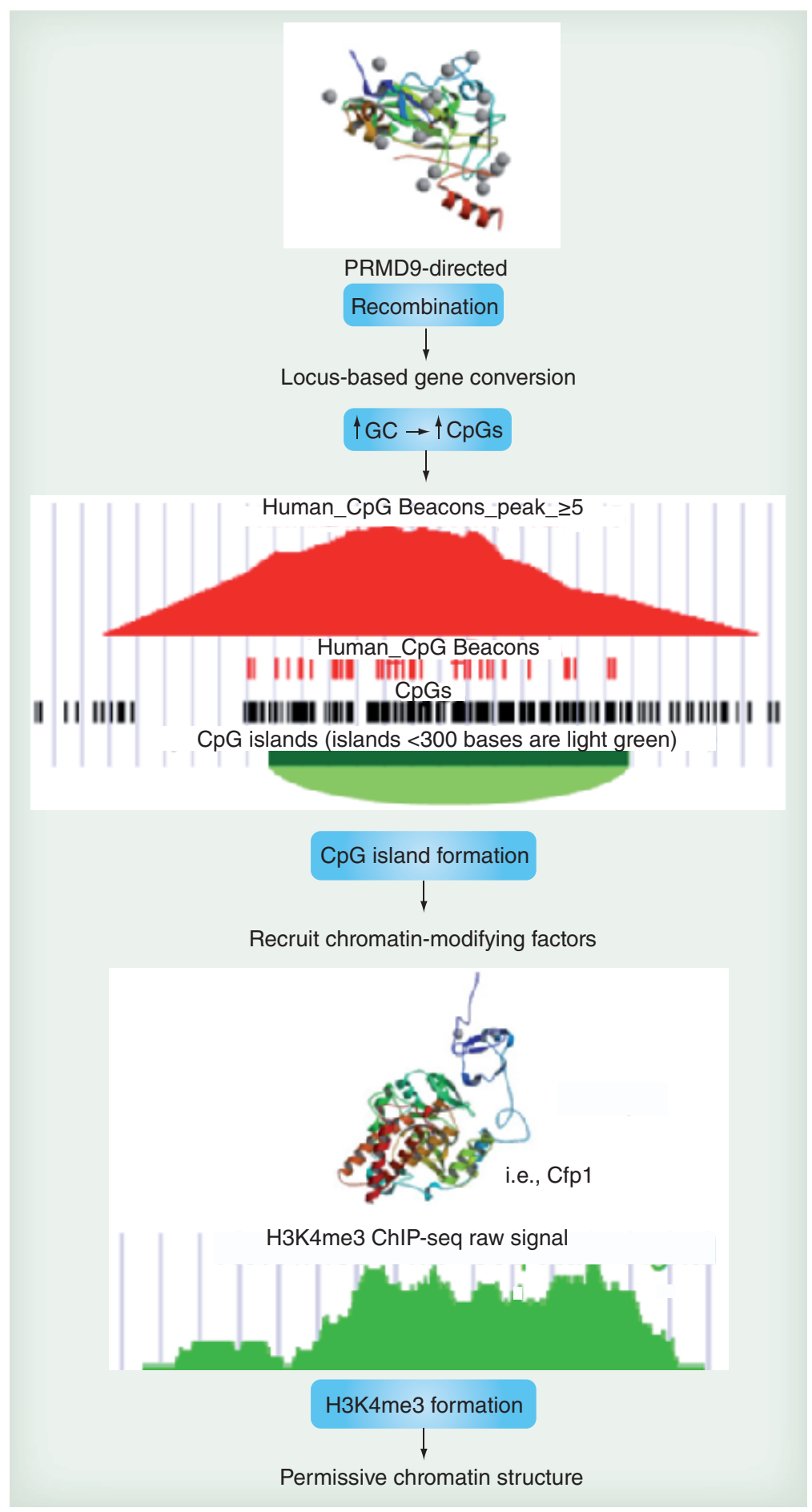

Figure 5. The hypothesis associating potential recombination-driven permissive chromatin formation with CPGi formation via CpG 'beacons'. The histone methylase PRDM9 directs recombination via motif-specificity, with resultant biased gene conversion within these regions increasing GC content and therefore $\mathrm{CpG}$ numbers. Dense $\mathrm{CpG}$ regions are able to recruit chromatin-modifying factors such as Cpf1, which lead to the formation of permissive H3K4me3 chromatin domains. Due to the strong association of PRDM9 with recombination [51], ancestral alleles of this rapidly evolving DNA-binding factor [52] are hypothesized to be implicated in this recombination-associated biased gene conversion [39]. human, within these loci, is not due to severe concurrent loss of CpGs in all of the other primates. Thus we have shown by our primate-specific comparison that these sequence-directed or associated epigenetic changes, identified in murine models, in fact occur in human, and furthermore within the significant brain tissue of the human PFC.

Comparing DNA sequence can identify humanspecific epigenomic variation that can increase the understanding of brain function unique to humans. Epigenetics complements genetics, and is not in conflict with it, justifying an integrated approach in genomic and epigenomic analyses for disease studies [59-61]. Structural effects on the genome through chromatin modification can in fact be deduced by direct sequence comparison, especially when the importance of species-specific CpGs 'beacons' in the formation of $\mathrm{H} 3 \mathrm{~K} 4 \mathrm{me} 3$ is acknowledged and the additional power of comparison between multiple closely-related primates is employed.

\section{Conclusion}

We have shown that CpG 'beacon' clusters can precisely locate hs-H3K4me3 and, furthermore, are more enriched in the strongest human-specific peaks. This supports our work that the most extreme CpG 'beacon' peaks map to tissue-invariant peaks of accessible H3K4me3 chromatin, specific only to the human genome, and implicated in the formation of the human phenotype [9].

\section{Future perspective}

The integration of genomic, epigenomic and transcriptomic sequence data will enable an increasingly comprehensive understanding of biology. Rapid advances in sequencing technology will speed this powerful combinatorial analysis and facilitate detailed exploration of the evolution of malignant, population and species-specific variation. Increased understanding of how genetic sequence change, such as in CpGs and transcription factor motifs, may be associated with active or passive epigenomic alterations will aid deciphering these data. Furthermore, through these methods, the identification of functional differences, between humans and other closely related primates, will help explain the human-specific phenotype and increase the understanding of human pathophysiology.

\section{Open access}

This article is distributed under the terms of the Creative Commons Attribution License 3.0 which permits any use, distribution, and reproduction in any medium, provided the original author(s) and the source are credited. To view a copy of the license, visit http://creativecommons.org/licenses/by/3.0/ 
Financial \& competing interests

disclosure

This work was supported by the Wellcome Trust (99148), EUFP7 projects EPIGENESYS (257082) and BLUEPRINT (282510), and a Royal Society Wolfson Research Merit Award to S Beck (WM100023). The authors have no other relevant affiliations or financial involvement with any organization or entity with a financial interest in or financial conflict with the subject matter or materials discussed in the manuscript apart from those disclosed.

No writing assistance was utilized in the production of this manuscript.

\section{Executive summary}

- We observed that genetic human-specific CpG (CpG 'beacon') clusters are enriched for human-specific H3K4me3 peaks identified in the prefrontal cortex.

- Therefore, these CpG 'beacon' clusters are predictive of the location of this permissive chromatin state within the human genome.

- The strongest human-specific H3K4me3 peaks (lowest FDR) have the strongest CpG 'beacon' association.

- There is also an association with recombination, as both human-specific H3K4me3 and CpG 'beacon' clusters are increased within distal or telomeric chromosome regions (terminal chromosome bands).

- The process of biased gene conversion is implicated in these dramatic CpG dinucleotide increases.

\section{References}

Papers of special note have been highlighted as:

- $\quad$ of interest

1 Blackledge NP, Klose R. CpG island chromatin: a platform for gene regulation. Epigenetics 6(2), 147-152 (2011).

2 Illingworth RS, Bird AP. CpG islands-'a rough guide'. FEBS Lett. 583(11), 1713-1720 (2009).

3 Thomson JP, Skene PJ, Selfridge J et al. CpG islands influence chromatin structure via the CpG-binding protein Cfp1. Nature 464(7291), 1082-1086 (2010).

- This publication identified that direct $\mathrm{CPG}$ inclusion could lead to the formation of $\mathrm{H} 3 \mathrm{~K} 4 \mathrm{me} 3$ in a mouse model.

4 Blackledge NP, Zhou JC, Tolstorukov MY, Farcas AM, Park PJ, Klose RJ. CpG islands recruit a histone H3 lysine 36 demethylase. Mol. Cell 38(2), 179-190 (2010).

5 Bird A. The dinucleotide CG as a genomic signalling module. J. Mol. Biol. 409(1), 47-53 (2011).

6 Kouzarides T. Chromatin modifications and their function. Cell 128(4), 693-705 (2007).

7 Guenther MG, Levine SS, Boyer LA, Jaenisch R, Young RA. A chromatin landmark and transcription initiation at most promoters in human cells. Cell 130(1), 77-88 (2007).

8 Vermeulen M, Mulder KW, Denissov S et al. Selective anchoring of TFIID to nucleosomes by trimethylation of histone H3 lysine 4. Cell 131(1), 58-69 (2007).

-9 Bell CG, Wilson GA, Butcher LM, Roos C, Walter L, Beck S. Human-specific CpG "beacons" identify loci associated with human-specific traits and disease. Epigenetics 7(10), 1188-1199 (2012).

- Details the identification of human-specific CpG 'beacons' via comparison with five other primates.

10 Paten B, Herrero J, Beal K, Fitzgerald S, Birney E. Enredo and Pecan: genome-wide mammalian consistency-based multiple alignment with paralogs. Genome Res. 18(11), 1814-1828 (2008).

11 Paten B, Herrero J, Fitzgerald S et al. Genome-wide nucleotide-level mammalian ancestor reconstruction. Genome Res. 18(11), 1829-1843 (2008).
- 12 Ziller MJ, Gu H, Muller F et al. Charting a dynamic DNA methylation landscape of the human genome. Nature 500, 477-481 (2013).

13 Sirmaci A, Spiliopoulos M, Brancati F et al. Mutations in ANKRD11 cause KBG syndrome, characterized by intellectual disability, skeletal malformations, and macrodontia. Am. J. Hum. Genet. 89(2), 289-294 (2011).

14 Frints SG, Marynen P, Hartmann D et al. CALL interrupted in a patient with non-specific mental retardation: gene dosage-dependent alteration of murine brain development and behavior. Hum. Mol. Genet. 12(13), 1463-1474 (2003).

Kleefstra T, Brunner HG, Amiel J et al. Loss-of-function mutations in euchromatin histone methyl transferase 1 (EHMT1) cause the 9q34 subtelomeric deletion syndrome. Am. J. Hum. Genet. 79(2), 370-377 (2006).

- 16 Boycott KM, Flavelle S, Bureau A et al. Homozygous deletion of the very low density lipoprotein receptor gene causes autosomal recessive cerebellar hypoplasia with cerebral gyral simplification. Am. J. Hum. Genet. 77(3), 477-483 (2005).

17 Trommsdorff M, Gotthardt M, Hiesberger T et al. Reeler/ Disabled-like disruption of neuronal migration in knockout mice lacking the VLDL receptor and ApoE receptor 2. Cell 97(6), 689-701 (1999).

18 Marshall CR, Noor A, Vincent JB et al. Structural variation of chromosomes in autism spectrum disorder. Am. J. Hum. Genet. 82(2), 477-488 (2008).

19 Pinto D, Pagnamenta AT, Klei L et al. Functional impact of global rare copy number variation in autism spectrum disorders. Nature 466(7304), 368-372 (2010).

-20 Djurovic S, Gustafsson O, Mattingsdal M et al. A genomewide association study of bipolar disorder in Norwegian individuals, followed by replication in Icelandic sample. J. Affect. Disord. 126(1-2), 312-316 (2010).

-21 Pollard KS, Salama SR, Lambert N et al. An RNA gene expressed during cortical development evolved rapidly in humans. Nature 443(7108), 167-172 (2006). 
-22 Eckhardt F, Lewin J, Cortese R et al. DNA methylation profiling of human chromosomes 6, 20 and 22. Nat. Genet. 38(12), 1378-1385 (2006).

-23 Weber M, Hellmann I, Stadler MB et al. Distribution, silencing potential and evolutionary impact of promoter DNA methylation in the human genome. Nat. Genet. 39(4), 457-466 (2007).

24 Shulha HP, Crisci JL, Reshetov D et al. Human-specific histone methylation signatures at transcription start sites in prefrontal neurons. PLoS Biol. 10(11), e1001427 (2012).

- Describes the identification of human-specific peaks from the comparative chromatin immunoprecipitation sequencing analysis of the prefrontal cortex of human, chimpanzee and macaque.

-25 Semendeferi K, Teffer K, Buxhoeveden DP et al. Spatial organization of neurons in the frontal pole sets humans apart from great apes. Cereb. Cortex 21(7), 1485-1497 (2011).

-26 Varki A, Geschwind DH, Eichler EE. Explaining human uniqueness: genome interactions with environment, behaviour and culture. Nat. Rev. Genet. 9(10), 749-763 (2008).

-27 Maunakea AK, Nagarajan RP, Bilenky M et al. Conserved role of intragenic DNA methylation in regulating alternative promoters. Nature 466(7303), 253-257 (2010).

28 NCBI SRA. Evolutionarily conserved role of intragenic DNA methylation in regulating alternative promoters. www.ncbi.nlm.nih.gov/Traces/sra/?study=SRP002318

29 UCL. Medical Genomics. www2.cancer.ucl.ac.uk/medicalgenomics/ humanCpGBeacons/trackList.php

- 30 Ihaka R, Gentleman R. R: a language for data analysis and graphics. J. Comp. Graph. Stat. 5(3), 299-314 (1996).

-31 Quinlan AR, Hall IM. BEDTools: a flexible suite of utilities for comparing genomic features. Bioinformatics 26(6), 841-842 (2010).

- 32 Krzywinski M, Schein J, Birol I et al. Circos: an information aesthetic for comparative genomics. Genome Res. 19(9), 1639-1645 (2009).

- 33 Mclean CY, Bristor D, Hiller M et al. GREAT improves functional interpretation of cis-regulatory regions. Nat. Biotechnol. 28(5), 495-501 (2010).

- 34 Bickel PJ, Boley N, Brown JB, Huang HY, Zhang NR. Subsampling methods for genomic inference. Ann. Appl. Stat. 4(4), 1660-1697 (2010).

- 35 Yip KY, Cheng C, Bhardwaj N et al. Classification of human genomic regions based on experimentally determined binding sites of more than 100 transcription-related factors. Genome Biol. 13(9), R48 (2012).

- 36 Sanyal A, Lajoie BR, Jain G, Dekker J. The long-range interaction landscape of gene promoters. Nature 489 (7414), 109-113 (2012).

- 37 Ernst J, Kellis M. ChromHMM: automating chromatin-state discovery and characterization. Nat. Methods 9(3), 215-216 (2012).

- 38 Dreszer TR, Wall GD, Haussler D, Pollard KS. Biased clustered substitutions in the human genome: the footprints of male-driven biased gene conversion. Genome Res. 17(10), 1420-1430 (2007).

- Identified that regions of extreme sequence divergence occur commonly within regions of biased gene conversion associated with recombination.

39 Capra JA, Hubisz MJ, Kostka D, Pollard KS, Siepel A. A model-based analysis of GC-biased gene conversion in the human and chimpanzee genomes. PLoS Genet. 9(8), e1003684 (2013).

-40 Fan Y, Newman T, Linardopoulou E, Trask BJ. Gene content and function of the ancestral chromosome fusion site in human chromosome 2q13-2q14.1 and paralogous regions. Genome Res. 12(11), 1663-1672 (2002).

-41 Cohen NM, Kenigsberg E, Tanay A. Primate CpG islands are maintained by heterogeneous evolutionary regimes involving minimal selection. Cell 145(5), 773-786 (2011).

42 Akey JM. Constructing genomic maps of positive selection in humans: where do we go from here? Genome Res. 19(5), 711-722 (2009).

43 Prabhakar S, Visel A, Akiyama JA et al. Human-specific gain of function in a developmental enhancer. Science 321(5894), 1346-1350 (2008).

44 Bird CP, Stranger BE, Liu M et al. Fast-evolving noncoding sequences in the human genome. Genome Biol. 8(6), R118 (2007).

- 45 Smith ZD, Meissner A. DNA methylation: roles in mammalian development. Nat. Rev. Genet. 14(3), 204-220 (2013).

46 Varley KE, Gertz J, Bowling KM et al. Dynamic DNA methylation across diverse human cell lines and tissues. Genome Res. 23(3), 555-567 (2013).

47 Branco MR, Ficz G, Reik W. Uncovering the role of 5-hydroxymethylcytosine in the epigenome. Nat. Rev. Genet. 13(1), 7-13 (2012)

48 Zarnack K, Konig J, Tajnik M et al. Direct competition between hnRNP C and U2AF65 protects the transcriptome from the exonization of Alu elements. Cell 152(3), 453-466 (2013).

- 49 Das R, Dimitrova N, Xuan Z et al. Computational prediction of methylation status in human genomic sequences. Proc. Natl Acad. Sci. USA 103(28), 10713-10716 (2006).

50 Myers S, Bowden R, Tumian A et al. Drive against hotspot motifs in primates implicates the PRDM9 gene in meiotic recombination. Science 327(5967), 876-879 (2010).

-51 Baudat F, Buard J, Grey C et al. PRDM9 is a major determinant of meiotic recombination hotspots in humans and mice. Science 327(5967), 836-840 (2010).

52 Oliver PL, Goodstadt L, Bayes JJ et al. Accelerated evolution of the Prdm9 speciation gene across diverse metazoan taxa. PLoS Genet. 5(12), e1000753 (2009).

53 Galtier N, Duret L. Adaptation or biased gene conversion? Extending the null hypothesis of molecular evolution. Trends Genet. 23(6), 273-277 (2007).

-54 Long HK, Sims D, Heger A et al. Epigenetic conservation at gene regulatory elements revealed by non-methylated DNA profiling in seven vertebrates. Elife 2, e00348 (2013). 
55 Polak P, Arndt PF. Long-range bidirectional strand asymmetries originate at $\mathrm{CpG}$ islands in the human genome. Genome Biol. Evol. 1, 189-197 (2009).

56 Clouaire T, Webb S, Skene P et al. Cfp1 integrates both CpG content and gene activity for accurate $\mathrm{H} 3 \mathrm{~K} 4 \mathrm{me} 3$ deposition in embryonic stem cells. Genes Dev. 26(15), 1714-1728 (2012).

57 Fenouil R, Cauchy P, Koch F et al. CpG islands and GC content dictate nucleosome depletion in a transcriptionindependent manner at mammalian promoters. Genome Res. 22(12), 2399-2408 (2012).

58 Yu A, Zhao C, Fan Y et al. Comparison of human genetic and sequence-based physical maps. Nature 409(6822), 951-953 (2001).
59 Birney E. Chromatin and heritability: how epigenetic studies can complement genetic approaches. Trends Genet. 27(5), 172-176 (2011)

Bell CG. Integration of genomic and epigenomic DNA methylation data in common complex diseases by haplotypespecific methylation analysis. Personalized Med. 8(3), 243-251 (2011).

61 Mattick JS. RNA driving the epigenetic bus. EMBO J. 31(3), 515-516 (2012). 